\title{
Partial Automata and Finitely Generated Congruences: An Extension of Nerode's Theorem*
}

\author{
Dexter Kozen \\ Computer Science Department \\ Cornell University \\ Ithaca, New York 14853-7501, USA \\ kozen@cs.cornell.edu
}

For Anil Nerode, on the occasion of his $60^{\text {th }}$ birthday

June 4, 1992

\begin{abstract}
Let $T_{\Sigma}$ be the set of ground terms over a finite ranked alphabet $\Sigma$. We define partial automata on $T_{\Sigma}$ and prove that the finitely generated congruences on $T_{\Sigma}$ are in one-to-one correspondence (up to isomorphism) with the finite partial automata on $T_{\Sigma}$ with no inaccessible and no inessential states. We give an application in term rewriting: every ground term rewrite system has a canonical equivalent system that can be constructed in polynomial time.
\end{abstract}

*in: Logical Methods: in Honor of Anil Nerode's Sixtieth Birthday, ed. J. N. Crossley, J. B. Remmel, R. A. Shore, and M. E. Sweedler, Birkhäuser, Boston, 1993, 490-511. 


\section{Introduction}

The Myhill-Nerode Theorem is a classic result in the theory of finite automata. It dates to work of Myhill [13] and Nerode [14] in the late 1950s, but is still today considered one of the most important results in the subject. It has numerous applications, especially in showing that certain sets are regular or certain apparently stronger types of automata are really no more powerful than finite automata. Nevertheless, its statement and proof are elementary enough that it can be taught in introductory courses.

The Myhill-Nerode Theorem exploits a fundamental connection between combinatorics and algebra to give a particularly satisfying characterization of the regular sets over a finite alphabet. As presented in a standard undergraduate text [9], it states:

Myhill-Nerode Theorem [13, 14] Let $R$ be a set of strings over a finite alphabet $\Sigma$. The following three propositions are equivalent:

(i) $R$ is accepted by a finite automaton

(ii) $R$ is a union of classes of a right-invariant equivalence relation of finite index

(iii) the relation $\equiv_{R}$ is of finite index, where $x \equiv_{R} y$ iff

$$
\forall z \in \Sigma^{*} x z \in R \leftrightarrow y z \in R
$$

The equivalence of (i) and (ii) is generally established using the following lemma:

Correspondence Lemma Up to isomorphism, there is a one-to-one correspondence between the right-invariant equivalence relations of finite index on $\Sigma$ and deterministic finite automata over $\Sigma$ with no inaccessible states.

Essentially, the states correspond to the equivalence classes, and the property of right invariance allows the deterministic transition function to be defined unambiguously on equivalence classes.

The Myhill-Nerode Theorem generalizes in a straightforward way to automata on finite trees. This generalization first came to light in the late 
1960s, ten years after Myhill and Nerode's work, and can be attributed to a combination of results of Brainerd [2, 3], Eilenberg and Wright [5], and Arbib and Give'on [1], although one must also credit Thatcher and Wright [17] in this context with the development of the algebraic approach to automata on finite trees, which allows "conventional finite automata theory [to go] through for the generalization-and... quite neatly" [17]. A particularly easy proof of this generalization in the style of [9] can be found in [12].

In the Thatcher-Wright approach to automata on finite trees, the elements of $\Sigma$ are assigned finite arities, and instead of strings one works with the ground terms $T_{\Sigma}$ over $\Sigma$. A deterministic finite tree automaton over $\Sigma$ is just a finite $\Sigma$-algebra $\mathcal{A}$, consisting of a finite carrier $|\mathcal{A}|$ and a distinguished $n$-ary function $f^{\mathcal{A}}:|\mathcal{A}|^{n} \rightarrow|\mathcal{A}|$ for each $n$-ary symbol $f \in \Sigma$. This definition includes the nullary case $(n=0)$, in which the function symbol is called a constant and interpreted as an element of $|\mathcal{A}|$. By analogy with the combinatorial treatment of $[9]$, we call elements of $|\mathcal{A}|$ states.

Since $T_{\Sigma}$ is the free $\Sigma$-algebra on the empty set of generators, there exists a unique $\Sigma$-algebra homomorphism

$$
\delta: T_{\Sigma} \rightarrow \mathcal{A}
$$

This map assigns a unique state $\delta(t)$ to each term $t$ in an inductive fashion, and is analogous to "running" the automaton on input $t$. A state is said to be accessible if it is $\delta(t)$ for some term $t$.

An equivalence relation $R$ on $T_{\Sigma}$ is said to be recognized by the automaton $\mathcal{A}$ if the kernel of $\delta$ (i.e., the relation $\{(s, t) \mid \delta(s)=\delta(t)\})$ refines $R$. In other words, $R$ is recognized by $\mathcal{A}$ if for any terms $s, t \in T_{\Sigma}$, if $\delta(s)=\delta(t)$, then $s R t$. The special case of regular sets discussed above corresponds to an $R$ with two equivalence classes, namely the regular set and its complement. If $R$ is recognized by $\mathcal{A}$, it is possible to partition the states of $\mathcal{A}$ such that the inverse image of the partition under $\delta$ coincides with the equivalence classes of $R$; this partition of the states corresponds to the specification of a set of final or accept states in the special case of regular sets.

For a given equivalence relation $R \subseteq T_{\Sigma}$ (recognizable or not), define $s \equiv_{R} t$ if for all terms $u$ with exactly one occurrence of a variable $x$ and no other variables,

$$
u[x / s] \quad R \quad u[x / t],
$$


where $u[x / s]$ denotes the term obtained by substituting $s$ for $x$ in $u$. The relation $\equiv_{R}$ generalizes the relation on strings of the same name mentioned above.

Myhill-Nerode Theorem for trees [3, 5, 1] Let $R$ be an equivalence relation on $T_{\Sigma}$. The following three propositions are equivalent:

(i) $R$ is recognizable

(ii) there exists a congruence on $T_{\Sigma}$ of finite index refining $R$

(iii) the relation $\equiv_{R}$ is of finite index.

The Myhill-Nerode theorem for strings corresponds to the special case of a single nullary operator and several unary operators.

In the algebraic approach, the tree version of the Correspondence Lemma reduces to an elementary fact of universal algebra: up to isomorphism, the homomorphic images of $T_{\Sigma}$ and the congruences on $T_{\Sigma}$ are in one-to-one correspondence. The correspondence is given by the quotient construction

$$
\equiv \mapsto T_{\Sigma} / \equiv
$$

in which it is readily observed that the quotient is finite iff the corresponding congruence is of finite index.

In $[10,11]$, we investigated the complexity of various decision problems in $\Sigma$-algebras presented by finite sets of ground equations over $T_{\Sigma}$; that is, quotients of $T_{\Sigma}$ modulo finitely generated congruences on $T_{\Sigma}$. We showed, among other results, that every such algebra has a minimal canonical presentation that is unique up to isomorphism.

This result has an interesting interpretation in terms of the Myhill-Nerode Theorem. First, we note that every congruence $\equiv$ on $T_{\Sigma}$ of finite index is finitely generated. To see this, let $U \subseteq T_{\Sigma}$ be a complete set of representatives for the $\equiv$-classes, and consider the finite subrelation consisting of all pairs in $\equiv$ of the form

$$
f u_{1} \ldots u_{n} \equiv u
$$

for $u_{1}, \ldots, u_{n}, u \in U$ and $f \in \Sigma_{n}$. The relation generated by the equations (1) is surely contained in $\equiv$; conversely, an easy inductive argument shows 
that every term is equivalent to the $u \in U$ in its $\equiv$-class under the congruence generated by the equations (1).

However, not every finitely generated congruence is of finite index: for example, the identity relation on $T_{\Sigma}$ is of infinite index (assuming $\Sigma$ has at least one constant and at least one symbol of higher arity), but is generated by the empty relation.

The question thus arises as to whether there is a more general version of the Myhill-Nerode theorem with "finitely generated" in place of "finite index".

The answer to this question is mixed. On the positive side, we formulate and prove a version of the Correspondence Lemma in this more general setting. On the other hand, we construct an equivalence relation $R$ that has no minimal refining finitely generated congruence.

In order to formulate the first result, we need a combinatorial structure that is to finitely generated congruences as finite tree automata are to congruences of finite index. The appropriate notion is a finite partial automaton on $T_{\Sigma}$. Simply stated, a finite partial automaton is just a finite partial $\Sigma$ algebra, where a partial $\Sigma$-algebra is like a $\Sigma$-algebra except the distinguished operations need not be everywhere defined. We will show how a finite partial automaton $\mathcal{A}$ uniquely determines a possibly infinite set of "states". This is done formally by a universal algebraic construction giving a certain total extension $\hat{\mathcal{A}}$ of $\mathcal{A}$ called its free total extension.

These results have an interesting application to term rewriting: every ground term rewrite system has a canonical equivalent system which is unambiguous and in which all rules are of the form $f q_{1} \ldots q_{n} \rightarrow q$, where $q_{1}, \ldots, q_{n}, q$ are auxiliary constants. By canonical we mean that the system is minimal and unique up to isomorphism. The canonical system can be obtained effectively from the original system in polynomial time. This gives a method for testing the equivalence of ground term rewrite systems over a finite signature in polynomial time.

A proof of the existence of the canonical system and a polynomial time algorithm for deriving it appeared in $[10,11]$ (Lemmas 24 and 25), although it was not stated in terms of term rewriting. Faster $O(n \log n)$ algorithms for this problem have recently been given by Snyder [16] and Fülöp and Vágvölgyi [7].

Partial algebras are discussed in [8]. Nondeterministic partial automata have been considered previously in [15]. Although the approach is new, many 
of the essential ideas behind the results of this paper are more or less implicit in $[10,11]$.

\section{Partial Algebras and Partial Automata}

Partial algebras, subalgebras, homomorphisms, and congruences are discussed in [8].

Let $\Sigma$ be an arbitrary but fixed finite ranked alphabet. The rank of $f \in \Sigma$ is called its arity. The set of $n$-ary elements of $\Sigma$ is denoted $\Sigma_{n}$. Nullary symbols $c \in \Sigma_{0}$ are called constants. We usually use $c, d, \ldots$ for constants and $f, g, \ldots$ for function symbols in $\Sigma$ of any arity. The set of ground terms over $\Sigma$ is denoted $T_{\Sigma}$. The depth of a term is the length of the longest path from the root to a leaf, thinking of terms as labeled trees.

Definition 2.1 A partial E-algebra (or just partial algebra for short) is a structure

$$
\mathcal{A}=\left(|\mathcal{A}|, \cdot^{\mathcal{A}}\right)
$$

where $|\mathcal{A}|$ is a set, called the carrier of $\mathcal{A}$, and $\cdot \mathcal{A}$ assigns a partial $n$-ary function

$$
f^{\mathcal{A}}:|\mathcal{A}|^{n} \rightarrow|\mathcal{A}|
$$

to each $n$-ary function symbol $f$ of $\Sigma$. By partial we mean that $f^{\mathcal{A}}$ need not be everywhere defined. We identify nullary functions

$$
c^{\mathcal{A}}:|\mathcal{A}|^{0} \rightarrow|\mathcal{A}|
$$

with elements of $|\mathcal{A}|$. Like functions of higher arity, $c^{\mathcal{A}}$ may be undefined.

The partial algebra $\mathcal{A}$ is said to be total if all functions $f^{\mathcal{A}}$ are everywhere defined. It is said to be finite if $|\mathcal{A}|$ is a finite set.

The set $T_{\Sigma \cup Q}$ of ground terms over the disjoint union $\Sigma \cup Q$, where $Q$ is a new set of constants, with operations

$$
f^{T_{\Sigma \cup Q}}\left(t_{1}, \ldots, t_{n}\right)=f t_{1} \ldots t_{n},
$$

is the free (total) $\Sigma$-algebra on generators $Q$. 
Definition 2.2 A congruence on a total $\Sigma$-algebra $\mathcal{A}$ is an equivalence relation $\equiv$ on $|\mathcal{A}|$ such that $f^{\mathcal{A}}\left(a_{1} \ldots a_{n}\right) \equiv f^{\mathcal{A}}\left(b_{1} \ldots b_{n}\right)$ whenever $f \in \Sigma_{n}$ and $a_{i} \equiv b_{i}, 1 \leq i \leq n$. If $\Gamma$ is a binary relation on $|\mathcal{A}|$, the congruence generated by $\Gamma$ is the smallest congruence on $|\mathcal{A}|$ containing $\Gamma$. For $a, b \in \mathcal{A}$, we write $a \equiv b(\Gamma)$ and say $a$ and $b$ are congruent modulo $\Gamma$ if $a$ and $b$ are equivalent modulo the congruence generated by $\Gamma$. A congruence $\equiv$ is finitely generated if it is generated by a finite subrelation.

An equivalence relation $\equiv$ is of finite index if there are only finitely many $\equiv$-classes. An equivalence relation $R$ refines another equivalence relation $S$ if each $S$-class is a union of $R$-classes; equivalently, if $s R t$ implies $s S t$.

Congruences on partial algebras are defined in [8], but for the purposes of this paper we will only need to consider congruences on $T_{\Sigma \cup Q}$.

Definition 2.3 Let $\mathcal{A}$ and $\mathcal{B}$ be two partial $\Sigma$-algebras. A (total) function

$$
h: \mathcal{A} \rightarrow \mathcal{B}
$$

is a partial $\Sigma$-algebra homomorphism (or just partial homomorphism for short) if, whenever $q_{1}, \ldots, q_{n} \in \mathcal{A}, f \in \Sigma_{n}$, and $f^{\mathcal{A}}\left(q_{1}, \ldots, q_{n}\right)$ is defined, then $f^{\mathcal{B}}\left(h\left(q_{1}\right), \ldots, h\left(q_{n}\right)\right)$ is defined and equal to $h\left(f^{\mathcal{A}}\left(q_{1}, \ldots, q_{n}\right)\right)$. We emphasize that partial homomorphisms are always total functions.

We write $\mathcal{A} \sqsubseteq \mathcal{B}$ and say that $\mathcal{A}$ is a partial subalgebra of $\mathcal{B}$ (or weak subalgebra in the terminology of [8]) and that $\mathcal{B}$ is an extension of $\mathcal{A}$ if $|\mathcal{A}| \subseteq|\mathcal{B}|$ and the inclusion map $\mathcal{A} \rightarrow \mathcal{B}$ is a partial homomorphism.

A partial subalgebra $\mathcal{A}$ of $\mathcal{B}$ is said to be the induced partial subalgebra of $\mathcal{B}$ on $Q \subseteq|\mathcal{B}|$ (or relative subalgebra in the terminology of [8]) if $|\mathcal{A}|=Q$ and for all $q_{1}, \ldots, q_{n} \in Q$ and $f \in \Sigma_{n}$,

$$
f^{\mathcal{A}}\left(q_{1}, \ldots, q_{n}\right)=f^{\mathcal{B}}\left(q_{1}, \ldots, q_{n}\right)
$$

whenever the right hand side is defined and in $Q$.

Definition 2.4 If $\mathcal{A}$ is a partial algebra, let $T_{\Sigma \cup|\mathcal{A}|}$ be the set of ground terms over the disjoint union $\Sigma \cup|\mathcal{A}|$. The binary relation

$$
\begin{aligned}
\Delta_{\mathcal{A}}=\left\{\left(q, f q_{1} \ldots q_{n}\right) \mid\right. & q_{1}, \ldots, q_{n}, q \in|\mathcal{A}|, f \in \Sigma_{n}, \\
& \left.f^{\mathcal{A}}\left(q_{1}, \ldots, q_{n}\right) \text { exists and is equal to } q\right\}
\end{aligned}
$$

is called the diagram of $\mathcal{A}$. 
The term partial automaton is synonymous with partial algebra. When thinking automata-theoretically, we often call elements of $|\mathcal{A}|$ states.

A conventional tree automaton over $\Sigma$ in the sense of Thatcher and Wright is just a finite total $\Sigma$-algebra $\mathcal{A}$. Informally, such an automaton takes a ground term in $T_{\Sigma}$ as input. It starts at the leaves and moves upward, associating a state with each subterm inductively. If the immediate subterms $t_{1}, \ldots, t_{n}$ of the term $f t_{1} \ldots t_{n}$ are labeled with states $q_{1}, \ldots, q_{n}$ respectively, then the term $f t_{1} \ldots t_{n}$ will be labeled with state $f^{\mathcal{A}}\left(q_{1}, \ldots, q_{n}\right)$. Note that the basis of the induction is included here: the state labeling the term $c$ is $c^{\mathcal{A}}$.

Formally, the labeling function is just the unique $\Sigma$-algebra homomorphism

$$
\delta: T_{\Sigma} \rightarrow \mathcal{A}
$$

from the free $\Sigma$-algebra $T_{\Sigma}$ to $\mathcal{A}$. By considerations of universal algebra, this homomorphism exists and is unique. A state of $\mathcal{A}$ is said to be accessible if it is in the image of $T_{\Sigma}$ under $\delta$, inaccessible otherwise. Thus we would say that the automaton $\mathcal{A}$ has no inaccessible states if the map $\delta$ is onto.

This definition extends the usual definition of automata on finite strings in a natural way: we can think of an automaton on strings over a finite alphabet $\Sigma$ as a tree automaton over $\Sigma \cup\{\square\}$, where $\square$ is a new constant and elements of $\Sigma$ are assigned arity 1 .

Equivalently, we can define tree automata as term rewrite systems. This is the approach taken for example in [6]. Given an algebra $\mathcal{A}$, we can consider $\Delta_{\mathcal{A}}$ as a ground term rewrite system on $T_{\Sigma \cup|\mathcal{A}|}$ in which the equations are ordered from right to left. This system is unambiguous (in the sense that there are no overlapping redexes) and terminating, thus normal forms exist and are unique [4]. By elementary considerations of term rewrite theory, the terms $s$ and $t$ are congruent modulo $\Delta_{\mathcal{A}}$ iff they have the same normal form. For a total algebra $\mathcal{A}$, the $\Delta_{\mathcal{A}}$-normal form of term $t$ is $\delta(t) \in|\mathcal{A}|$. For an intruduction to term rewrite theory, see [4].

\section{$3 \quad$ Free Total Extensions}

A partial automaton runs inductively on a ground term in the same way as a total automaton. However, the reader is probably already asking the obvious 
question: what happens when it reaches a situation from which it cannot continue because the appropriate $f^{\mathcal{A}}\left(q_{1}, \ldots, q_{n}\right)$ is undefined? Informally, whenever it encounters such a situation, it creates a new state symbolically and moves to it. In this way a finite partial automaton $\mathcal{A}$ gives rise to a possibly infinite set $\hat{\mathcal{A}}$ of symbolic states that could be created in this way. The construction of $\hat{\mathcal{A}}$ from $\mathcal{A}$ is analogous to the construction of algebraic extensions of fields or of the rational numbers from the integers, where we wish to extend the structure in the freest possible way so that certain functions are defined. We formalize this idea by the notion of free total extension of a partial algebra.

Formally, free total extensions are defined in terms of their most salient property, a universality property similar to that of free algebras.

Definition 3.1 A total extension $\hat{\mathcal{A}}$ of a partial algebra $\mathcal{A}$ is a free total extension if for any total algebra $\mathcal{B}$ and partial $\Sigma$-algebra homomorphism $h: \mathcal{A} \rightarrow \mathcal{B}$, there is a unique $\Sigma$-algebra homomorphism $\widehat{h}: \widehat{\mathcal{A}} \rightarrow \mathcal{B}$ such that the diagram

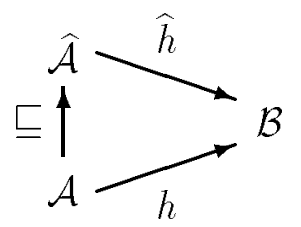

commutes.

Theorem 3.2 Free total extensions exist and are unique up to isomorphism. Moreover, a partial algebra $\mathcal{A}$ is the induced partial subalgebra of its free total extension $\hat{\mathcal{A}}$ on $|\mathcal{A}|$.

Proof. Let $\Delta_{\mathcal{A}}$ be the diagram of $\mathcal{A}$ (Definition 2.4) and take $\hat{\mathcal{A}}=$ $T_{\Sigma \cup|\mathcal{A}|} / \Delta_{\mathcal{A}}$. Let $\nu(t)$ denote the $\Delta_{\mathcal{A}}$-normal form of $t \in T_{\Sigma \cup|\mathcal{A}|}$, and let $[t]$ denote the congruence class of $t$ modulo $\Delta_{\mathcal{A}}$. The canonical map $t \mapsto[t]$ restricted to domain $|\mathcal{A}|$ constitutes a partial homomorphism $\mathcal{A} \rightarrow \widehat{\mathcal{A}}$, since if $f^{\mathcal{A}}\left(q_{1}, \ldots, q_{n}\right)=q$, then $q \equiv f q_{1} \ldots q_{n} \in \Delta_{\mathcal{A}}$, therefore

$$
f^{\widehat{\mathcal{A}}}\left(\left[q_{1}\right], \ldots,\left[q_{n}\right]\right)=\left[f q_{1} \ldots q_{n}\right]=[q] .
$$

This map is also one-to-one on $\mathcal{A}$, since distinct elements of $\mathcal{A}$ have distinct normal forms $(\nu(q)=q$ for $q \in|\mathcal{A}|)$, therefore occupy distinct $\Delta_{\mathcal{A}}$-congruence classes. By a slight abuse, we may thus consider $\mathcal{A} \sqsubseteq \hat{\mathcal{A}}$. 
The partial algebra $\mathcal{A}$ is the induced partial subalgebra of $\widehat{\mathcal{A}}$ on $|\mathcal{A}|$, since if (3) holds with $q_{1}, \ldots, q_{n}, q \in \mathcal{A}$, then

$$
\nu\left(f q_{1} \ldots q_{n}\right)=\nu(q)=q
$$

thus $q \equiv f q_{1} \ldots q_{n} \in \Delta_{\mathcal{A}}$, therefore $f^{\mathcal{A}}\left(q_{1}, \ldots, q_{n}\right)$ exists and is equal to $q$.

If $h: \mathcal{A} \rightarrow \mathcal{B}$ is a partial $\Sigma$-algebra homomorphism from $\mathcal{A}$ to any total algebra $\mathcal{B}$, then let $h^{\prime}$ denote the unique homomorphism $T_{\Sigma \cup|\mathcal{A}|} \rightarrow \mathcal{B}$ such that $h^{\prime}(q)=h(q)$ for $q \in|\mathcal{A}|$. We wish to show that $h^{\prime}$ factors through $\hat{\mathcal{A}}$, giving the following commutative diagram:

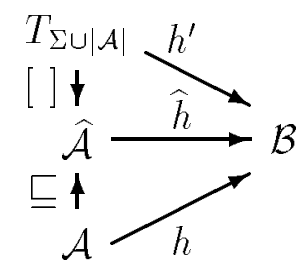

For this purpose it suffices to show that if $s \equiv t\left(\Delta_{\mathcal{A}}\right)$ then $h^{\prime}(s)=h^{\prime}(t)$. For any equation $q \equiv f q_{1} \ldots q_{n} \in \Delta_{\mathcal{A}}$, we have that $f^{\mathcal{A}}\left(q_{1}, \ldots, q_{n}\right)$ exists and is equal to $q$. Then

$$
\begin{aligned}
h^{\prime}(q) & =h(q) \\
& =h\left(f^{\mathcal{A}}\left(q_{1}, \ldots, q_{n}\right)\right) \\
& =f^{\mathcal{B}}\left(h\left(q_{1}\right), \ldots, h\left(q_{n}\right)\right) \\
& =f^{\mathcal{B}}\left(h^{\prime}\left(q_{1}\right), \ldots, h^{\prime}\left(q_{n}\right)\right) \\
& =h^{\prime}\left(f q_{1} \ldots q_{n}\right) .
\end{aligned}
$$

Since $\Delta_{\mathcal{A}}$ is contained in the kernel of $h^{\prime}$, so is the congruence generated by $\Delta_{\mathcal{A}}$. Thus $s \equiv t\left(\Delta_{\mathcal{A}}\right)$ implies $h^{\prime}(s)=h^{\prime}(t)$, and we have a unique map $\widehat{h}: \widehat{\mathcal{A}} \rightarrow \mathcal{B}$ that agrees with $h$ on $\mathcal{A}$.

The uniqueness of $\hat{\mathcal{A}}$ up to isomorphism follows directly from the universality property (2): if $\hat{\mathcal{A}}$ and $\hat{\mathcal{A}}^{\prime}$ are two free total extensions of $\mathcal{A}$, then there are unique homomorphisms between $\widehat{\mathcal{A}}$ and $\hat{\mathcal{A}}^{\prime}$ in either direction, and these must be inverses.

We have actually shown that the construction $\mathcal{A} \mapsto \hat{\mathcal{A}}$ constitutes a left adjoint to the inclusion functor from the category of total $\Sigma$-algebras and $\Sigma$-algebra homomorphisms to the category of partial $\Sigma$-algebras and partial ¿-algebra homomorphisms. 


\section{Essential Elements}

To get a one-to-one correspondence in the Correspondence Lemma, we had to delete inaccessible states from the automaton. We will have to do that here as well, but we will also have to delete other states that are inessential for the construction of the free total extension.

Intuitively, an element of a total $\Sigma$-algebra $\mathcal{A}$ is essential if it is a source of nonfreeness. For example, $q$ is essential if $q=f^{\mathcal{A}}(p)=g^{\mathcal{A}}(r)$ and $f \neq g$, or if $q=f^{\mathcal{A}}(q)$. This will imply that $q$ must be contained in any partial subalgebra of $\mathcal{A}$ having $\mathcal{A}$ as its free total extension. Moreover, we will show that under a mild restriction on how $\mathcal{A}$ is generated, the induced partial subalgebra of $\mathcal{A}$ on the set of its essential elements has $\mathcal{A}$ as its free total extension. Thus the induced partial subalgebra on the essential elements of $\mathcal{A}$ is the unique minimal partial subalgebra of $\mathcal{A}$ having $\mathcal{A}$ as its free total extension.

A unary function $|\mathcal{A}| \rightarrow|\mathcal{A}|$ is said to be definable (in $\mathcal{A}$ ) if it is of the form $\lambda x . t$, where $x \notin \Sigma$ is a nullary variable, $t$ is a term over $\Sigma \cup\{x\}$, and the function symbols $f \in \Sigma$ occurring in $t$ are interpreted as $f^{\mathcal{A}}$.

Definition 4.1 Let $\mathcal{A}$ be a total $\Sigma$-algebra. An element $q \in \mathcal{A}$ is said to be essential if any of the following five conditions hold:

(i) $q \neq f^{\mathcal{A}}\left(q_{1}, \ldots, q_{n}\right)$ for any $n \geq 0, f \in \Sigma_{n}$ and $q_{1}, \ldots, q_{n} \in \mathcal{A}$

(ii) $q=f^{\mathcal{A}}\left(p_{1}, \ldots, p_{m}\right)=g^{\mathcal{A}}\left(q_{1}, \ldots, q_{n}\right)$ and $f \neq g$

(iii) $q=f^{\mathcal{A}}\left(p_{1}, \ldots, p_{n}\right)=f^{\mathcal{A}}\left(q_{1}, \ldots, q_{n}\right)$ and $p_{i} \neq q_{i}$ for some $i, 1 \leq i \leq n$

(iv) $q=F(q)$ for some definable unary function $F=\lambda x . t$ on $\mathcal{A}$, and $t \neq x$

(v) $p=F(q)$ for some definable unary function $F$ on $\mathcal{A}$ and $p$ is essential. (Note that the definition is inductive because of this clause.)

We define $\mathcal{E} \mathcal{A}$ to be the induced partial subalgebra of $\mathcal{A}$ on the set of essential elements of $\mathcal{A}$. The partial algebra $\mathcal{E} \mathcal{A}$ is called the essential subalgebra of $\mathcal{A}$. An element of a partial algebra $\mathcal{A}$ is said to be essential if it is an essential element of $\hat{\mathcal{A}}$. (This definition does not conflict if $\mathcal{A}$ is total, since in this case $\widehat{\mathcal{A}} \cong \mathcal{A}$.) 
Definition 4.2 Let $\mathcal{A}$ be a total $\Sigma$-algebra. A subset $Q \subseteq|\mathcal{A}|$ is a generating set if the canonical map $T_{\Sigma \cup Q} \rightarrow \mathcal{A}$ is onto. The set $Q$ is a minimal generating set if it is a generating set and no subset of $Q$ is a generating set.

If $\mathcal{A}$ is a partial algebra, then the null set is a generating set of $\hat{\mathcal{A}}$ exactly when there are no inaccessible elements of $\mathcal{A}$, i.e., when the canonical map $T_{\Sigma} \rightarrow \widehat{\mathcal{A}}$ is onto. Of course, in this case the null set is also a minimal generating set. Any algebra with a finite generating set has a minimal generating set. The integers with successor give an example of an algebra with no minimal generating set.

Lemma 4.3 Let $\mathcal{A}$ be a total $\Sigma$-algebra possessing a minimal generating set $Q$. Then every element of $Q$ is essential.

Proof. Let

$$
\delta: T_{\Sigma \cup Q} \rightarrow \mathcal{A}
$$

be the canonical map in which $\delta(q)=q$ for $q \in Q$. For any $q \in Q$, if the only term $t \in T_{\Sigma \cup Q}$ with $q=\delta(t)$ is $q$ itself, then $q$ is essential by Definition 4.1(i). Otherwise, there exists an $n$-ary function symbol $f$ for some $n \geq 0$ and terms $t_{1}, \ldots, t_{n} \in T_{\Sigma \cup Q}$ such that $q=\delta\left(f t_{1} \ldots t_{n}\right)$. If $q$ occurs in some term $t_{i}$, then $q$ is essential by Definition 4.1(iv). If not, then $Q-\{q\}$ is a generating set, contradicting the assumption that $Q$ was minimal.

The next theorem justifies the term "essential". It shows that the essential elements of a total algebra $\mathcal{B}$ must be contained in any partial subalgebra having $\mathcal{B}$ as its free total extension.

Theorem 4.4 Any partial algebra $\mathcal{A}$ contains all essential elements of $\hat{\mathcal{A}}$. Moreover, the partial algebra $\mathcal{E} \hat{\mathcal{A}}$ is the induced partial subalgebra of $\mathcal{A}$ on the set of essential elements of $\hat{\mathcal{A}}$.

Proof. Let $\mathcal{E}=\mathcal{E} \hat{\mathcal{A}}$, let $t \mapsto[t]$ be the canonical map $T_{\Sigma \cup|\mathcal{A}|} \rightarrow \widehat{\mathcal{A}}$, and let $\nu(t)$ denote the $\Delta_{\mathcal{A}}$-normal form of $t \in T_{\Sigma \cup|\mathcal{A}|}$. We show first that $|\mathcal{E}| \subseteq|\mathcal{A}|$. For any $e \in|\mathcal{E}|$, let $t \in T_{\Sigma \cup|\mathcal{A}|}$ be the unique term in $\Delta_{\mathcal{A}}$-normal form with $[t]=e$. 
If $e \in|\mathcal{E}|$ because of Definition 4.1(i), then $t$ must be $e$ itself. Thus $e \in|\mathcal{A}|$.

If $e \in|\mathcal{E}|$ because of Definition 4.1(ii), then there exist terms $f s_{1} \ldots s_{m}$ and $g t_{1} \ldots t_{n}$ with

$$
\nu\left(f s_{1} \ldots s_{m}\right)=\nu\left(g t_{1} \ldots t_{n}\right)=t .
$$

Since these two terms have distinct head symbols but the same normal form, $t$ must coincide with $e$, therefore $e \in|\mathcal{A}|$.

If $e \in|\mathcal{E}|$ because of Definition 4.1(iii), then there exist terms $f s_{1} \ldots s_{n}$ and $f t_{1} \ldots t_{n}$ with

$$
\nu\left(f s_{1} \ldots s_{n}\right)=\nu\left(f t_{1} \ldots t_{n}\right)=t
$$

but

$$
\nu\left(s_{i}\right) \neq \nu\left(t_{i}\right)
$$

for some $i, 1 \leq i \leq n$. Again, in order for $f s_{1} \ldots s_{n}$ and $f t_{1} \ldots t_{n}$ to have the same normal form, $t$ must coincide with $e$ and $e \in|\mathcal{A}|$.

If $e \in|\mathcal{E}|$ because of Definition 4.1(iv), then there exists a term $s$ with exactly one occurrence of a variable $x$, but not $x$ itself, such that

$$
\nu(s[x / t])=t .
$$

Since $s$ is not $x$ itself, the depth of $s[x / t]$ is strictly greater than the depth of $t$. In order to reduce $s[x / t]$ to $t$, since $t$ is in normal form, the occurrence of $t$ in $s[x / t]$ must be an element of $|\mathcal{A}|$, and this element must be $e$.

Finally, if $e \in|\mathcal{E}|$ because of Definition 4.1(v), then there exists a term $s$ with one occurrence of a variable $x$ such that $s[x / t] \equiv p\left(\Delta_{\mathcal{A}}\right)$ and $p$ is essential. By the induction hypothesis, $p \in|\mathcal{A}|$, so $\nu(s[x / t])=p$. Therefore the occurrence of $t$ in $s[x / t]$ must be an element of $|\mathcal{A}|$, and this element must be $e$.

We have shown that $|\mathcal{E}| \subseteq|\mathcal{A}|$. Since $\mathcal{E}$ is the induced partial subalgebra of $\hat{\mathcal{A}}$ on $|\mathcal{E}|$ and $\mathcal{A}$ is the induced partial subalgebra of $\hat{\mathcal{A}}$ on $|\mathcal{A}|$ (Theorem 3.2), it follows that the inclusion map $\mathcal{E} \rightarrow \mathcal{A}$ is a partial $\Sigma$-algebra homomorphism and that $\mathcal{E}$ is the induced partial subalgebra of $\mathcal{A}$ on $|\mathcal{E}|$.

Theorem 4.5 Let $\mathcal{A}$ be a total $\Sigma$-algebra with essential subalgebra $\mathcal{E}=\mathcal{E} \mathcal{A}$. Then $\hat{\mathcal{E}}$ is embedded isomorphically in $\mathcal{A}$. Moreover, if $\mathcal{A}$ contains a minimal generating set, then $\widehat{\mathcal{E}}$ and $\mathcal{A}$ are isomorphic. 
Proof. By definition, $\mathcal{E} \sqsubseteq \mathcal{A}$. By Theorem 3.2, there exists a unique homomorphism $h: \hat{\mathcal{E}} \rightarrow \mathcal{A}$ with $h$ the identity on $\mathcal{E}$. We wish to show that $h$ is injective.

Let $h^{\prime}: T_{\Sigma \cup|\mathcal{E}|} \rightarrow \mathcal{A}$ be the canonical map with $h^{\prime}(q)=q$ for $q \in|\mathcal{E}|$. We have the following commutative diagram:

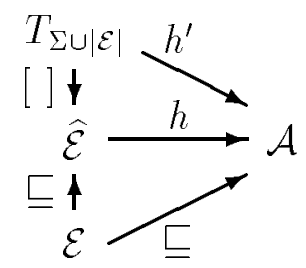

We wish to show that for any $s, t \in T_{\Sigma \cup|\mathcal{E}|}$, if $h^{\prime}(s)=h^{\prime}(t)$ then $s \equiv t\left(\Delta_{\mathcal{E}}\right)$.

We show first that if $t \in T_{\Sigma \cup|\mathcal{E}|}$ is in $\Delta_{\mathcal{E}}$-normal form and $h^{\prime}(t)=q \in|\mathcal{E}|$, then $t=q$. Suppose for a contradiction that $t=f t_{1} \ldots t_{n}, f \in \Sigma_{n}$, and $t$ is

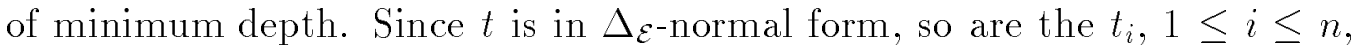
and

$$
\begin{aligned}
q & =h^{\prime}\left(f t_{1} \ldots t_{n}\right) \\
& =f^{\mathcal{A}}\left(h^{\prime}\left(t_{1}\right), \ldots, h^{\prime}\left(t_{n}\right)\right) .
\end{aligned}
$$

By Definition $4.1(\mathrm{v}), h^{\prime}\left(t_{i}\right) \in|\mathcal{E}|$, say $h^{\prime}\left(t_{i}\right)=q_{i}$. Since $t$ was of minimum depth, $t_{i}=q_{i}, 1 \leq i \leq n$. We thus have

$$
q=f^{\mathcal{A}}\left(q_{1}, \ldots, q_{n}\right),
$$

thus

$$
q \equiv f q_{1} \ldots q_{n} \in \Delta_{\mathcal{E}}
$$

contradicting the assumption that $t$ was in normal form.

Now let $s, t \in T_{\Sigma \cup|\mathcal{E}|}$ be in $\Delta_{\mathcal{E}^{\text {-normal }}}$ form, and suppose $h^{\prime}(s)=h^{\prime}(t)$. We proceed by induction on the form of $s$ and $t$.

If $s=q \in|\mathcal{E}|$, then $h^{\prime}(s)=h^{\prime}(t)=q$, thus $s=t=q$. The argument is similar for $t \in|\mathcal{E}|$. Otherwise, assume neither $s$ nor $t$ is in $|\mathcal{E}|$.

If $s=f s_{1} \ldots s_{m}$ and $t=g t_{1} \ldots t_{n}$ and $f \neq g$, then

$$
\begin{aligned}
f^{\mathcal{A}}\left(h^{\prime}\left(s_{1}\right), \ldots, h^{\prime}\left(s_{m}\right)\right) & =h^{\prime}\left(f s_{1} \ldots s_{m}\right) \\
& =h^{\prime}\left(g t_{1} \ldots t_{n}\right) \\
& =g^{\mathcal{A}}\left(h^{\prime}\left(t_{1}\right), \ldots, h^{\prime}\left(t_{n}\right)\right)
\end{aligned}
$$


and $h^{\prime}(s) \in|\mathcal{E}|$ by Definition 4.1(ii), contradicting the assumption that $h^{\prime}(s) \notin|\mathcal{E}|$.

If $s=f s_{1} \ldots s_{n}$ and $t=f t_{1} \ldots t_{n}$, and if some $h^{\prime}\left(s_{i}\right) \neq h^{\prime}\left(t_{i}\right)$, then we obtain a contradiction as in the previous case, using Definition 4.1(iii).

Thus we are left with the case $s=f s_{1} \ldots s_{n}, t=f t_{1} \ldots t_{n}$, and $h^{\prime}\left(s_{i}\right)=$ $h^{\prime}\left(t_{i}\right), 1 \leq i \leq n$. By the induction hypothesis, $s_{i} \equiv t_{i}\left(\Delta_{\mathcal{E}}\right), 1 \leq i \leq n$, therefore $s \equiv t\left(\Delta_{\mathcal{E}}\right)$.

If $\mathcal{A}$ contains a minimal generating set $Q$, then $Q \subseteq \mathcal{E}$ by Lemma 4.3, thus $\mathcal{E}$ is also a generating set. Since $\mathcal{E}$ also generates $\overline{\mathcal{E}}$, the map $h$ is onto in this case.

Corollary 4.6 Let $\mathcal{A}$ be a total $\Sigma$-algebra possessing a minimal generating set. Up to isomorphism, the essential subalgebra $\mathcal{E} \mathcal{A}$ of $\mathcal{A}$ is the unique minimal partial algebra having free total extension $\mathcal{A}$.

The corollary is not true in general for algebras not possessing a minimal generating set. For example, consider a nonstandard model of the natural numbers with 0 and successor and the usual Peano axioms over this signature. There is no minimal set generating the nonstandard elements, and there are no essential elements. Thus the free total extension of the essential subalgebra consists of the standard natural numbers.

\section{$5 \quad$ Partial Automata and Finitely Generated Congruences}

The following theorem is our version of the Correspondence Lemma generalized to partial automata and finitely generated congruences.

Theorem 5.1 Up to isomorphism, there is a one-to-one correspondence between (finitely generated) congruences on $T_{\Sigma}$ and (finite) partial automata over $T_{\Sigma}$ with no inaccessible and no inessential states.

Proof. We establish a one-to-one correspondence between congruences on $T_{\Sigma}$ and partial $\Sigma$-algebras with no inaccessible and no inessential elements, and show that a congruence is finitely generated iff its corresponding partial algebra is finite. 
For a congruence $\equiv$ on $T_{\Sigma}$, let $\mathcal{E}=\mathcal{E}\left(T_{\Sigma} / \equiv\right)$ be the essential subalgebra of the quotient $T_{\Sigma} / \equiv$. Since the canonical map $T_{\Sigma} \rightarrow T_{\Sigma} / \equiv$ is onto, $T_{\Sigma} / \equiv$ has minimal generating set $\emptyset$. By Theorem 4.5,

$$
\widehat{\mathcal{E}} \cong T_{\Sigma} / \equiv \text {, }
$$

therefore $\mathcal{E}$ has no inessential or inaccessible elements. Thus the map

$$
\equiv \mapsto \mathcal{E}\left(T_{\Sigma} / \equiv\right)
$$

takes congruences on $T_{\Sigma}$ to partial $\Sigma$-algebras with no inaccessible and no inessential elements.

Conversely, let $\mathcal{A}$ be a partial $\Sigma$-algebra with no inaccessible and no inessential elements, and let $\sim_{\mathcal{A}}$ be the kernel of the canonical map $\delta: T_{\Sigma} \rightarrow$ $\hat{\mathcal{A}}$. This construction gives a map

$$
\mathcal{A} \mapsto \sim \mathcal{A}
$$

from partial $\Sigma$-algebras with no inaccessible and no inessential elements to congruences on $T_{\Sigma}$.

We now show that the maps (4) and (5) are inverses up to isomorphism. For any congruence $\equiv$ on $T_{\Sigma}$, let $\mathcal{E}=\mathcal{E}\left(T_{\Sigma} / \equiv\right)$. Then $\equiv$ and $\sim_{\mathcal{E}}$ are the same relation, since $\delta$ is the unique homomorphism

$$
\delta: T_{\Sigma} \rightarrow \widehat{\mathcal{E}} \cong T_{\Sigma} / \equiv
$$

Conversely, for any partial $\Sigma$-algebra $\mathcal{A}$ with no inaccessible or inessential elements, we wish to show that $\mathcal{A}$ and $\mathcal{E}=\mathcal{E}\left(T_{\Sigma} / \sim_{\mathcal{A}}\right)$ are isomorphic. We have by Theorem 4.4 that $\mathcal{E} \hat{\mathcal{A}}$ is the induced partial subalgebra of $\mathcal{A}$ on $|\mathcal{E}|$. Since $\mathcal{A}$ has no inessential elements,

$$
\mathcal{A} \cong \mathcal{E} \hat{\mathcal{A}}
$$

Since $\mathcal{A}$ has no inaccessible elements, the canonical map $\delta: T_{\Sigma} \rightarrow \widehat{\mathcal{A}}$ is onto, thus

$$
\widehat{\mathcal{A}} \cong T_{\Sigma} / \sim_{\mathcal{A}}
$$

therefore

$$
\mathcal{E} \hat{\mathcal{A}} \cong \mathcal{E}\left(T_{\Sigma} / \sim_{\mathcal{A}}\right)
$$

Finally, we show 
(i) if $\mathcal{A}$ is finite, then $\sim_{\mathcal{A}}$ is finitely generated

(ii) if $\Gamma$ is a finite relation on $T_{\Sigma}$, then $\mathcal{E}\left(T_{\Sigma} / \Gamma\right)$ is finite.

First (i). If $\mathcal{A}$ is finite, then so is $\Delta_{\mathcal{A}}$. Since $\delta: T_{\Sigma} \rightarrow \hat{\mathcal{A}}$ is onto, for each $q \in|\mathcal{A}|$ there exists an $\eta(q) \in T_{\Sigma}$ such that $\delta(\eta(q))=[q]$. The map $\eta$ extends uniquely to a homomorphism $\eta: T_{\Sigma \cup|\mathcal{A}|} \rightarrow T_{\Sigma}$, and by uniqueness of the maps we have that the diagram

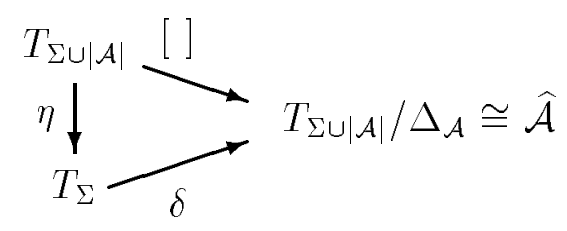

commutes. Thus for $s, t \in T_{\Sigma \cup|\mathcal{A}|}$,

$$
\begin{aligned}
s \equiv t\left(\Delta_{\mathcal{A}}\right) & \leftrightarrow[s]=[t] \\
& \leftrightarrow \delta(\eta(s))=\delta(\eta(t)) \\
& \leftrightarrow \eta(s) \sim_{\mathcal{A}} \eta(t) .
\end{aligned}
$$

We now show that $\sim_{\mathcal{A}}$ is generated by the finite relation

$$
\eta\left(\Delta_{\mathcal{A}}\right)=\left\{(\eta(s), \eta(t)) \mid(s, t) \in \Delta_{\mathcal{A}}\right\}
$$

on $T_{\Sigma}$. Certainly the congruence on $T_{\Sigma}$ generated by $\eta\left(\Delta_{\mathcal{A}}\right)$ is contained in $\sim_{\mathcal{A}}$ since $\eta\left(\Delta_{\mathcal{A}}\right)$ is, and a straightforward inductive argument on the length of derivations in $\Delta_{\mathcal{A}}$ shows that for any $s, t \in T_{\Sigma \cup|\mathcal{A}|}$,

$$
s \equiv t\left(\Delta_{\mathcal{A}}\right) \rightarrow \eta(s) \equiv \eta(t)\left(\eta\left(\Delta_{\mathcal{A}}\right)\right) .
$$

In particular, for $s, t \in T_{\Sigma}$, we have $s=\eta(s)$ and $t=\eta(t)$, thus

$$
\begin{aligned}
s \sim_{\mathcal{A}} t & \leftrightarrow s \equiv t\left(\Delta_{\mathcal{A}}\right) \\
& \leftrightarrow s \equiv t\left(\eta\left(\Delta_{\mathcal{A}}\right)\right) .
\end{aligned}
$$

To show (ii), let $\Gamma$ be a finite relation on $T_{\Sigma}$. Define a finite partial $\Sigma$ algebra $\mathcal{A}$ as follows. Let $t \mapsto[t]$ be the canonical map $T_{\Sigma} \rightarrow T_{\Sigma} / \Gamma$. Call the 
term $t$ present in $\Gamma$ if $t$ is a subterm of some $u$ or $v$ appearing in an equation $u \equiv v \in \Gamma$. Let $\mathcal{A}$ be the induced partial subalgebra of $T_{\Sigma} / \Gamma$ on the set

$$
\{[t] \mid t \text { is present in } \Gamma\} \text {. }
$$

By Theorem 3.2, the inclusion map $\mathcal{A} \rightarrow T_{\Sigma} / \Gamma$ extends uniquely to a homomorphism $h: \hat{\mathcal{A}} \rightarrow T_{\Sigma} / \Gamma$. Let $\delta$ be the canonical map $T_{\Sigma} \rightarrow \hat{\mathcal{A}}$. We have the commutative diagram

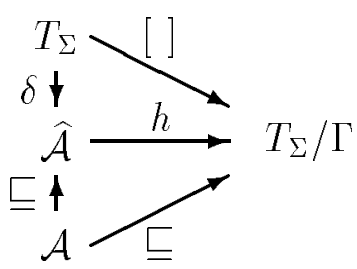

We show that $h$ is an isomorphism. It is certainly onto, since [ ] is. To show that it is one-to-one, it suffices to show that $\delta$ is onto and for $s, t \in T_{\Sigma}$, $s \equiv t(\Gamma)$ implies $\delta(s)=\delta(t)$.

A straightforward inductive argument shows that $\delta(t)=[t]$ for $t$ present in $\Gamma$ : if $f t_{1} \ldots t_{n}$ is present in $\Gamma$, then

$$
\left[f t_{1} \ldots t_{n}\right] \equiv f\left[t_{1}\right] \ldots\left[t_{n}\right] \in \Delta_{\mathcal{A}},
$$

therefore

$$
\begin{aligned}
\delta\left(f t_{1} \ldots t_{n}\right) & =f^{\widehat{\mathcal{A}}}\left(\delta\left(t_{1}\right), \ldots, \delta\left(t_{n}\right)\right) \\
& =f^{\hat{\mathcal{A}}}\left(\left[t_{1}\right], \ldots,\left[t_{n}\right]\right) \\
& =\left[f t_{1} \ldots t_{n}\right] .
\end{aligned}
$$

Since $\widehat{\mathcal{A}}$ is generated by $|\mathcal{A}|, \delta$ is onto. Now if $s \equiv t \in \Gamma$, then $[s]=[t] \in|\mathcal{A}|$, and $\delta(s)=\delta(t)=[s]$. Since the relation $\Gamma$ is contained in the kernel of $\delta$, so is the congruence generated by $\Gamma$. Thus $s \equiv t(\Gamma)$ implies $\delta(s)=\delta(t)$.

By Theorem 4.4, the essential subalgebra $\mathcal{E}\left(T_{\Sigma} / \Gamma\right)$ is contained in $\mathcal{A}$ and is therefore finite.

The following theorem was essentially proved in [10] and [11, Lemma 25], to which we refer the reader for the algorithm and proof of correctness.

Theorem $5.2([10,11])$ Given any finite relation $\Gamma$ on $T_{\Sigma}$, the diagram $\Delta_{\mathcal{E}}$ of $\mathcal{E}=\mathcal{E}\left(T_{\Sigma} / \Gamma\right)$ can be produced from $\Gamma$ in polynomial time.

By Corollary $4.6, \Delta_{\mathcal{E}}$ gives a canonical presentation of the finitely presented algebra $T_{\Sigma} / \Gamma$. 


\section{A Counterexample}

Let $R$ be an equivalence relation on $T_{\Sigma}$. Although the relation $\equiv_{R}$ is always the coarsest congruence on $T_{\Sigma}$ refining $R$, it need not be finitely generated, even if $R$ has only two classes. We construct such an $R$ below. Since there always exists a finitely generated congruence refining $R$ (namely the identity), the analog of clause (iii) in the statement of the Myhill-Nerode Theorem fails for partial automata.

Let $\Sigma=\{c, f, g\}$, where $c$ is nullary and $f, g$ are unary. (We need at least two unary symbols or one binary symbol to construct a counterexample, since all congruences over an alphabet with one unary symbol and no symbols of higher arity are finitely generated.)

Let $|t|$ denote the depth of term $t$. Let $A \subseteq \mathcal{N}$ be the set of powers of 2 (or any nonregular set of natural numbers). Define

$$
R=\{t|| t \mid \in A \text { and the head symbol of } t \text { is } f\} .
$$

Then $s \equiv_{R} t$ iff either

- $|s|=|t|$ and $|s| \notin A$, or

- $|s|=|t|,|s| \in A$, and the head symbols of $s$ and $t$ agree.

The quotient $T_{\Sigma} / \equiv_{R}$ looks like this:

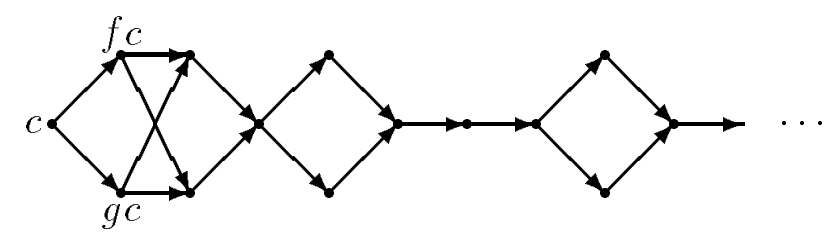

The congruence $\equiv_{R}$ is not finitely generated, since any finite subrelation of $\equiv_{R}$ is contained in the congruence generated by some $\equiv_{R}^{n}$, where $s \equiv_{R}^{n} t$ iff $|s|=|t| \leq n$ and $s \equiv_{R} t$. The quotient $T_{\Sigma} / \equiv_{R}^{n}$ is a strict homomorphic preimage of $T_{\Sigma} / \equiv_{R}$. It looks like this for $n=6$ :

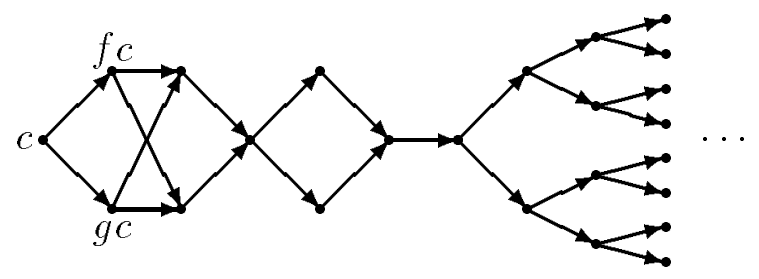




\section{Applications to Term Rewrite Systems}

Theorems 5.1 and 5.2 have the following application to term rewrite systems. Suppose we are given a ground term rewrite system over $\Sigma$. Let $Q$ be a new set of auxiliary constants disjoint from $\Sigma$. Let us call a ground term rewrite system over $\Sigma \cup Q$ simple if

- all rules are of the form $f q_{1} \ldots q_{n} \rightarrow q$, where $q_{1}, \ldots, q_{n}, q \in Q$ and $f \in \Sigma_{n}$

- the system is unambiguous in the sense that there are no overlapping redexes.

A system over $\Sigma \cup Q$ is said to be equivalent to the original system over $\Sigma$ if they induce the same congruence on $T_{\Sigma}$.

Theorems 5.1 and 5.2 have the following interpretation in this context:

Corollary 7.1 For every ground term rewrite system $\Gamma$ over $\Sigma$, there is a unique minimal simple system $\Gamma^{\prime}$ equivalent to $\Gamma$. Moreover, $\Gamma^{\prime}$ can be constructed from $\Gamma$ in polynomial time.

The system $\Gamma^{\prime}$ is of course just $\Delta_{\mathcal{E}}$, where $\mathcal{E}$ is the essential subalgebra of $T_{\Sigma} / \Gamma$.

This corollary appears in $[10,11]$ (Lemmas 24 and 25), although not stated in the language of term rewrite systems. Improved algorithms have recently been obtained by Snyder [16] and Fülöp and Vágvölgyi [7]. These algorithms run in time $O(n \log n)$ and are the fastest known algorithms for this problem.

It is decidable in polynomial time whether two $\Sigma$-algebras presented by finite sets of ground equations over $\Sigma$ are isomorphic [10, 11]: one tests

whether all the defining equations of one presentation are consequences of the defining equations of the other presentation, and vice versa. Corollary 4.6 gives an alternative method: construct the essential subalgebras and test whether they are isomorphic.

\section{Acknowledgements}

I am grateful to Wayne Snyder and an anonymous referee for references and valuable comments on an earlier version of this paper. 
This research was done while the author was on sabbatical at Aarhus University, Denmark. Support from the Danish Research Academy, the National Science Foundation under grant CCR-8806096, the John Simon Guggenheim Foundation, and the U.S. Army Research Office through the ACSyAM branch of the Mathematical Sciences Institute of Cornell University, contract DAAL03-91-C-0027 is gratefully acknowledged.

\section{References}

[1] M. A. Arbib and Y. Give'on. Algebra automata I: parallel programming as a prolegomenon to the categorical approach. Infor. and Control, 12:331-345, 1968.

[2] W. S. Brainerd. Tree Generating Systems and Tree Automata. PhD thesis, Purdue University, 1967.

[3] W. S. Brainerd. The minimalization of tree automata. Infor. and Control, 13:484-491, 1968.

[4] N. Dershowitz and J.-P. Jouannaud. Rewrite systems. In J. van Leeuwen, editor, Handbook of Theoretical Computer Science, volume B, pages 243-320. North Holland, 1990.

[5] S. Eilenberg and J. B. Wright. Automata in general algebra. Infor. and Control, 11:452-470, 1967.

[6] Z. Fülöp and S. Vágvölgyi. Congruential tree languages are the same as recognizable tree languages - a proof for a theorem of D. Kozen. Bull. Europ. Assoc. Theor. Comput. Sci., 39:175-184, October 1989.

[7] Z. Fülöp and S. Vágvölgyi. Ground term rewriting rules for the word problem of ground term equations. Bull. Europ. Assoc. Theor. Comput. Sci., 45:186201, October 1991.

[8] G. Grätzer. Universal Algebra. Springer-Verlag, second edition, 1979.

[9] J. E. Hopcroft and J. D. Ullman. Introduction to Automata Theory, Languages, and Computation. Addison-Wesley, 1979.

[10] D. Kozen. Complexity of Finitely Presented Algebras. PhD thesis, Cornell University, May 1977. 
[11] D. Kozen. Complexity of finitely presented algebras. In Proc. 9th ACM Symp. Theory of Comput., pages 164-177, May 1977.

[12] D. Kozen. On the Myhill-Nerode theorem for trees. Bull. Europ. Assoc. Theor. Comput. Sci., 47:170-173, June 1992.

[13] J. Myhill. Finite automata and the representation of events. Technical Report 57-264, WADC, 1957.

[14] A. Nerode. Linear automata transformations. Proc. Amer. Math. Soc., 9:541$544,1958$.

[15] A. Nerode, A. Yakhnis, and V. Yakhnis. Concurrent programs as strategies in games. In Y. Moschovakis, editor, Logic from Computer Science. SpringerVerlag, 1992.

[16] W. Snyder. Fast ground completion: an $O(n \log n)$ algorithm for generating reduced sets of ground rewrite rules equivalent to a set of ground equations. In Nachum Dershowitz, editor, Proc. Third Int. Conf. Rewriting Techniques and Applications, pages 419-433. Springer-Verlag Lect. Notes in Comput. Sci. $355,1989$.

[17] J. W. Thatcher and J. B. Wright. Generalized finite automata theory with an application to a decision problem of second order logic. Math. Syst. Theory, $2: 57-81,1968$. 